Culture et démocratie : réflexions à partir de la polémique entre libéraux taiwanais et néoconfucéens contemporains

Culture and Democracy. Reflections Based on the Debates between Taiwan's

Liberals and Contemporary New Confucians

現代新儒家與臺灣自由派的論戰及其意義

\title{
Ming-huei Lee
}

Traducteur : Sébastien Billioud

\section{OpenEdition Journals}

Édition électronique

URL : http://journals.openedition.org/extremeorient/129

DOI : 10.4000/extremeorient. 129

ISSN : 2108-7105

\section{Éditeur}

Presses universitaires de Vincennes

\section{Édition imprimée}

Date de publication : 1 octobre 2009

Pagination : 33-62

ISBN : 978-2-84292-237-5

ISSN : 0754-5010

\section{Référence électronique}

Ming-huei Lee, «Culture et démocratie : réflexions à partir de la polémique entre libéraux taiwanais et néo-confucéens contemporains », Extrême-Orient Extrême-Occident [En ligne], 31 | 2009, mis en ligne le 01 octobre 2012, consulté le 19 avril 2019. URL : http://journals.openedition.org/extremeorient/129 ; DOI : 10.4000/extremeorient.129 


\title{
Culture et démocratie: réflexions à partir de la polémique entre libéraux taiwanais et néo-confucéens contemporains
}

\author{
Lee Ming-huei \\ Traduction du chinois et notes de Sébastien Billioud
}

\section{Position du problème}

La démocratie, considérée dans son acception actuelle, tout comme le libéralisme, sont les produits de la culture occidentale moderne même si les libéraux occidentaux ne cessent d'en affirmer la valeur universelle. Au début $\mathrm{du} \mathrm{xx}^{\mathrm{e}}$ siècle, alors que ces idées se diffusaient en Chine, les libéraux chinois crurent eux aussi en leur valeur universelle. Un problème se posa alors naturellement: existait-il des contradictions fondamentales et indépassables entre cette universalité et les particularités de la tradition culturelle chinoise ? Que l'on quitte l'Occident pour l'Inde, l'Extrême-Orient ou encore le monde musulman, on réalise à quel point la question est loin de n'être que théorique. On ne peut, en réalité, en faire l'économie.

Dès le début, les libéraux chinois considérèrent que la tradition culturelle chinoise, incarnée au premier chef par le confucianisme ${ }^{1}$, constituait un obstacle de taille à la modernisation du pays tout comme à sa démocratisation. À cet égard, leur position était identique à celle des marxistes. En 1919, alors que la Première guerre mondiale venait de s'achever, l'opposition des étudiants à l'attribution au Japon de l'ancienne colonie allemande de Qingdao prévue par le traité de paix de Paris déboucha sur le célèbre Mouvement anti-gouvernemental du 4 mai. Ce dernier en généra immédiatement un autre, dit de la Nouvelle culture, fondamentalement opposé au confucianisme et porteur de slogans radicaux comme l'invitation à «abattre la boutique de Confucius». Les deux leaders de ce mouvement, et ce n'est pas une coïncidence, étaient Chen Duxiu

1. L'auteur évoque ici la façon dont le confucianisme, dans les années 1910 et 1920, en vint souvent et sans nuance, à être taxé de tous les maux et rendu responsable de l'arriération du pays. Il n'évoque pas un courant précis de la tradition confucéenne. N.d.T. 
(1879-1942), l'un des fondateurs du Parti communiste et Hu Shi (1891-1962), le héraut de la première génération de libéraux chinois.

Face à cette vague anti-traditionaliste extrême apparurent ceux que l'on appelle les néo-confucéens contemporains, dont la première génération fut incarnée par des figures comme Xiong Shili (1885-1968) et Liang Shuming (1893-1988 $)^{2}$. Même si les positions politiques de ces penseurs ne sont pas nécessairement homogènes, ils se sont tous accordés à dire que la Chine devait emprunter la voie de la démocratie. À cet égard, ils se distinguaient nettement d'autres confucéens de courants beaucoup plus conservateurs, comme celui de Kang Youwei (1858-1927) qui prônait une monarchie constitutionnelle et l'établissement du confucianisme comme religion d'État (guojiao). Néanmoins, ces «confucéens contemporains » soulignaient avec force que le processus de modernisation de la Chine (qui devait donc comprendre sa démocratisation) pouvait parfaitement s'accommoder de divers moyens pour préserver l'essence de la tradition confucéenne. À l'époque donc, dans le monde intellectuel chinois, le néo-confucianisme, le marxisme et libéralisme étaient les trois courants intellectuels les plus influents.

En 1949, le Parti communiste s'empara de la Chine continentale par la force et le Kuomintang fut contraint de se replier à Taiwan. L'île fut alors entraînée dans la guerre froide. Le marxisme devint sur le continent l'unique idéologie, tandis que le néo-confucianisme et le libéralisme furent confinés à Taiwan et Hong Kong. Ces deux courants créèrent des revues pour y exprimer leurs idées: Tribune démocratique (Minzhu Pinglun) pour le premier et Chine libre (Ziyou Zhongguo) pour le second. Comme ces deux publications s'opposaient au communisme, elles reçurent toutes deux le soutien financier du Kuomintang. Cependant, leur ligne éditoriale ne pouvait être pleinement contrôlée par ce dernier. Chine libre en arriva d'ailleurs à une rupture ouverte avec le Kuomintang, laquelle mena à l'interdiction de la revue.

En janvier 1958, à l'initiative de Tang Junyi qui en avait rédigé une première version, quatre intellectuels (Tang Junyi, Mou Zongsan, Zhang Junmai et Xu Fuguan) publièrent dans deux revues, Tribune démocratique et Renaissance

2. Les chercheurs et professeurs occidentaux ont en général l'habitude d'appeler «néoconfucéens » des penseurs des dynasties Song et Ming. Pour bien marquer la différence, j'emploie donc ici l'expression de «néo-confucéens contemporains». Savoir qui regrouper sous cette appellation a donné lieu à de multiples débats. Je pense pour ma part qu'il faut au moins intégrer huit personnages : en dehors de Xiong Shili et de Liang Shuming, ce groupe compte également Zhang Junmai (1887-1968), aussi connu en Occident sous le nom de Carsun Chang), Feng Youlan (1895-1990), Qian Mu (1895-1990), Tang Junyi (1909-1978), Mou Zongsan (1909-1995). Voir Lee Ming-huei, Der Konfuzianismus im modernen China, Leipzig, Leipziger Universitätsverlag, 2001, p.12 sq. 
(Zaisheng), un Manifeste en faveur de la culture chinoise adressé respectueusement aux citoyens du monde ${ }^{3}$. Ils y affirmaient que la Chine n'avait pas, dans le passé, généré de tradition scientifique et démocratique dans l'acception moderne, mais que ces deux éléments n'en étaient pas moins des «exigences intrinsèques» (neizai yaoqiu) d'une culture traditionnelle chinoise dans laquelle le confucianisme constituait la pièce centrale.

Dans un article précédent, $\mathrm{j}$ 'ai résumé les grands points de convergence et de divergence de ces trois grands courants - néo-confucianisme, marxisme et libéralisme:

Les libéraux et les néo-confucéens insistaient sur le fait que la Chine devait adopter un système démocratique parlementaire de type occidental. Les communistes taxaient au contraire ce dernier de «démocratie bourgeoise» en prônant pour leur part une «démocratie nouvelle» (xin minzhu). Cependant, libéraux et communistes adoptaient une position identique à l'égard de la culture traditionnelle chinoise, la considérant comme un obstacle à la modernisation. Au contraire, les confucéens non seulement ne la voyaient nullement incompatible avec la science et la démocratie, mais ils incorporaient aussi ces deux éléments dans ses «exigences intrinsèques». Ils reprochaient aux libéraux de ne considérer la science et la démocratie que comme des éléments importés quand eux les voyaient comme le chemin devant être emprunté par la nation chinoise pour développer ses idéaux culturels consciemment (zijue de), en tant que sujet spirituel (jingshen zhuti). [...] Face à ces arguments, les libéraux étaient des plus dubitatifs ${ }^{4}$.

\section{La polémique entre confucéens contemporains et libéraux à Taiwan}

Tant pour les libéraux que pour le confucéens, la chute de la Chine continentale aux mains des communistes était à la fois un drame et un rude coup porté

3. Ce manifeste a été publié dans Minzhu Pinglun (Tribune démocratique), vol. 9/1 (1 ${ }^{\mathrm{er}}$ mai 1958) et dans Zaisheng (Renaissance), vol. 1/1, janvier 1958. Il a ensuite été intégré dans Zhang Junmai, Cheng Wenxi (éd.), Zhong xi yin zhexue wenji (Recueil d'articles sur les philosophies chinoise, occidentale et indienne), Taibei, Taiwan xuesheng shuju, 1981 et dans Zhang Junmai, Xin Rujia sixiangshi (Histoire intellectuelle du néo-confucianisme contemporain), Taibei, Fondation pour la bourse Zhang Junmai, 1980. Sous le nom La Culture chinoise et le monde, le manifeste fut aussi publié dans Tang Junyi, Tang Junyi Quanji (Euvres complètes), Taibei, Taiwan xuesheng shuju, 1991, vol. 4. Une version anglaise de ce texte est également disponible dans Tang Junyi Quanji (Euvres complètes), op. cit., vol. 9 , p. 492-562.

4. Lee Ming-huei, Ruхие yи xiandai yishi (Confucianisme et conscience contemporaine), Taibei, Wenjin chubanshe, 1991, p. 1-2. La traduction est adaptée pour éviter des répétitions. N.d.T. 
au processus de modernisation du pays. Aussi, les deux camps partageaient un dessein bien réel : promouvoir les valeurs de la démocratie libérale et s'opposer à l'expansion communiste. Cet intérêt commun stimulait l'envie de collaborer. Il y eut dès lors des échanges, et les revues susmentionnées ouvrirent leurs colonnes à l'adversaire.

Progressivement, les différences entre les deux camps devinrent néanmoins de plus en plus manifestes, jusqu'à provoquer une polémique ouverte. Le détonateur en fut une critique de Mou Zongsan à l'endroit du célèbre logicien Jin Yuelin (1895-1984), resté sur le continent, et dont il avait d'ailleurs suivi les cours alors qu'il étudiait à l'université de Pékin dans les années 1929-1933. Avec l'arrivée des communistes, Jin, tout comme nombre d'autres intellectuels, fut contraint de se livrer à des séances d'autocritique. Après avoir eu vent du contenu de l'une d'entre elles, Mou écrivit le 2 janvier 1952 un essai dans la revue de Hong Kong L'homme libre, dont le titre était précisément: «Un homme véritablement libre ${ }^{5}$. Son idée centrale était la suivante : les libéraux de l'époque s'étaient, dans le domaine de la pensée, limités à des réflexions très techniques portant sur l'analyse logique et ils en étaient par conséquent devenus incapables d'affirmer quoi que ce soit sur l'individu (gexing) ou les valeurs. Cela se traduisait par une incapacité totale à s'opposer à l'oppression du Parti communiste et à porter les valeurs du libéralisme. Plus encore, cela les menait même à embrasser le matérialisme. Yin Haiguang (1919-1969), un ancien élève lui aussi de Jin Yuelin et éditeur de Chine libre, décida, à la lecture du texte de Mou Zongsan, de réagir. Sous le nom de plume de Mei Yunli, il publia un texte intitulé «L'homme véritablement libre que je connais » ${ }^{6}$, critiquant sévèrement la position de Mou, d'une part pour son manque de compréhension à l'égard des intellectuels restés sur le continent et, d'autre part, pour son «monisme idéaliste» (weixinlun de yiyuanlun). Son article se terminait de la sorte: «À travers tous les propos de Mou Zongsan on voit que le fond de sa pensée, à l'égard de la culture occidentale, n'est qu'une attitude de rejet. Je ne discerne aucune grande différence entre sa position et celle de ceux qui estimaient que le savoir chinois

5. Mou Zongsan, «Shidai yu ganshou xubian», in Mou Zongsan xiansheng quanji, Taibei, Lianjing chuban gongsi, 2003, vol. 24, p. 49-58.

6. Édité par Lin Zhenghong. Ce texte n'a pas été intégré dans les Euvres complètes de Yin Haiguang publiées par Taibei Guiguan tushu gongsi. Peut-être était-ce parce que l'éditeur ne connaissait pas le nom de plume utilisé par Yin Haiguang. La raison pour laquelle je peux avancer qu'il s'agit bien d'un texte de Yin Haiguang est que Xu Fuguan fait allusion à cela dans son article : «Ruhe fuhuo 'qiezhong shibi de taolun jingshen'», Minzhu Pinglun, vol. 6/9, 5 mai 1955. 
devait demeurer le fondement de tout le système tandis que le savoir occidental pouvait simplement être adopté pour sa dimension pratique ${ }^{7}$.»

L'année suivante, un autre intellectuel libéral, Zhang Foquan (1907-1994), publia successivement dans Chine libre et Tribune démocratique trois articles sur le concept de liberté, qui se voulaient aussi des critiques de la conception qu'en avaient les confucéens contemporains. Les deux camps se replièrent alors sur leurs positions, chacun publiant dans ses revues, jusqu'à aboutir à la rupture ouverte. Les protagonistes de cette polémique allaient être Xu Fuguan pour les confucéens et Yin Haiguang et Zhang Foquan du côté des libéraux.

Évoquer en détail l'histoire de cette polémique n'est pas ici notre objectif ${ }^{8}$. Nous cherchons plutôt à comparer et analyser les arguments des deux camps. Pour résumer, ceux-ci portaient d'abord sur deux points : (1) Dans quelle mesure la culture traditionnelle chinoise constituait-elle un obstacle à l'établissement d'un système démocratique et au développement de la science? Autrement dit, dans quelle mesure fallait-il d'abord y renoncer? (2) Une politique démocratique avait-t-elle besoin d'une base morale ? Une telle question revient à se demander dans quelle mesure la liberté morale (la libre volonté) doit être un préalable à la liberté politique.

Quelle était, sur le premier point, la position des confucéens contemporains? Tout développement nouveau, dans un domaine qui touche à la culture, doit s'enraciner dans la tradition. Des éléments exogènes ne peuvent donc être transposés directement, mais doivent au contraire être incorporés au développement intrinsèque de la tradition d'accueil, en s'ajustant avec elle. Nous avons déjà évoqué le discours contraire des libéraux pour lesquels la culture chinoise (du moins dans ses éléments centraux) constituait un obstacle à la modernisation. Pour eux, dès lors, ce projet confucéen d'ouverture vers la science et la démocratie à partir de la culture traditionnelle chinoise était dépourvu de toute base réaliste. Tout au plus le réduisaient-ils à une espèce de besoin psychologique d'autodéfense. Ils allaient même plus loin en estimant que la culture

7. Ibid., p. 13. Littéralement, il s'agit là de la formule zhongxue wei ti, xixue wei yong: «le savoir chinois comme fondement, le savoir occidental comme pratique». Elle était utilisée dans la seconde partie du XIX ${ }^{\mathrm{e}}$ siècle par des lettrés comme Zhang Zhidong qui, tout en étant partisans de la modernisation du pays (notamment sur un plan technologique, industriel) demeuraient conservateurs sur le plan des mœurs et valeurs fondamentales (confucianisme) ou en ce qui concerne le système politique. N.d.T.

8. Sur l'ensemble de cette polémique, voir mon article «Xu Fuguan yu Yin Haiguang» (Xu Fuguan et Yin Haiguang), in Lee Ming-huei, Dangdai Ruxue de ziwo zhuanhua, Taibei, Zhongyang yanjiuyuan Zhongguo wenzhe yanjiusuo, 1994, p. 89-127 et, pour une version en caractères simplifiés, Beijing, Zhongguo shehui kexue chubanshe, 2001, p. $81-117$. 
traditionnelle chinoise n'avait rien réussi à produire d'autre qu'une autocratie et que les confucéens contemporains, en la promouvant, ne se distinguaient guère des chantres de l'absolutisme.

Sur le second point, les confucéens estimaient qu'une culture politique démocratique devait être développée sur la base d'un idéalisme moral et que, par conséquent, la liberté politique requérait comme préliminaire la liberté morale $^{9}$. Ils admettaient certes une séparation entre les sphères politique et morale, mais estimaient que celles-ci n'étaient pas sans lien. De même, ils reconnaissaient les différences entre liberté politique et liberté morale et le fait que préconiser simplement cette dernière n'était nullement suffisant pour établir un système politique démocratique. Mais ils refusaient néanmoins de rompre avec ce socle moral et de se baser simplement sur un plan politique pour prôner les droits de l'homme et la liberté. En effet, dans leur esprit, un tel libéralisme était simplement sans racines, insuffisant pour relever le défi d'un communisme se réclamant avec force d'un idéal. De surcroît, les confucéens soutenaient aussi que pour affirmer un idéal moral et des valeurs, il n'était pas possible de se cantonner au monde empirique, mais qu'il fallait se référer à un monde transcendant et donc en fin de compte à une théorie métaphysique. Dès lors, la plupart des grands représentants du confucianisme contemporain ont élaboré un système métaphysique, ou du moins ont reconnu le sens et la valeur de la métaphysique. Ainsi, parmi les quatre signataires du Manifeste de 1958 évoqué plus haut, trois d'entre eux (Tang Junyi, Mou Zongsan et Zhang Junmai) ont été fortement influencés par l'idéalisme allemand, de Kant à Hegel ${ }^{10}$. Ce n'est évidemment pas fortuit.

Les libéraux taiwanais ont pour leur part hérité de la tradition libérale anglosaxonne, et en même temps des conceptions empiristes. L'idée de liberté qu'ils affirment est celle qu'Isaiah Berlin (1909-1997) qualifie de «négative » (xiaoji

9. Les confucéens contemporains évoqués par Lee Ming-huei ont tous été fortement influencés par l'idéalisme allemand, et notamment par Kant et Hegel. Ils ont largement utilisé leurs concepts tout en les réinterprétant. Au risque de simplifier des pensées complexes et qui ont de surcroît toutes leurs spécificités, disons que la liberté morale, chez les confucéens contemporains, tend vers une exigence de transformation morale de soi. Dès lors, ancrer la liberté politique dans la liberté morale revient à formuler une exigence vis-à-vis de l'individu qui va bien au-delà de la reconnaissance d'une «liberté négative». N.d.T.

10. Tang Junyi se tournait plutôt vers Hegel, Mou Zongsan et Zhang Junmai vers Kant. Sur l'influence de la philosophie de Kant sur Mou Zongsan et Zhang Junmai, voir : Li Mingui, Der Konfuzianismus im modernen China, Leipzig, Leipziger Universitätsverlag, chap. 8; Lee Ming-huei, «Kants Philosophie im modernen China», in Monika SchmitzEmans (éd.), Transkulturelle Rezeption und Konstruktion. Festschrift für Adrian Hsia, Heidelberg, Synchron Wissenschaftsverlag der Autoren, 2004, p. 30-37. 
ziyou, negative liberty). Dans ce sens, la liberté politique indique «le domaine au sein duquel un homme peut agir sans en être empêché par les autres ${ }^{11}$. Pour les confucéens, cette liberté est naturellement très importante, mais son établissement présuppose un autre type de liberté, celle que Berlin décrit comme «positive» (jiji ziyou, positive liberty). Cette dernière repose sur une conception de l'individu qui prend possession de lui-même et se régit sans recours à d'autres forces extérieures ${ }^{12}$. Pour dire les choses simplement, la «liberté négative» renvoie principalement à une liberté d'agir; la «liberté positive» touche quant à elle la liberté de notre volonté et revêt une dimension morale.

La raison pour laquelle I. Berlin a ainsi promu la liberté négative et condamné la liberté positive est que tant le nazisme que le fascisme ou le communisme n'ont eu cesse de se réclamer d'idéaux «moraux» (la gloire de la nation, la société communiste, etc.) pour servir leur contrôle despotique. Dans le même esprit que Berlin, les libéraux taiwanais se sont opposés à ce concept de liberté positive pour évoquer la liberté politique ou les droits de l'Homme. Pourquoi ? La liberté négative ne s'intéresse qu'à des actions et reste neutre sur le plan des valeurs. À partir de «libertés fondamentales» ou d'une «liste de droits», on peut en cerner clairement les contours et il n'est pas facile de la détourner. Au contraire, la liberté positive, en s'intéressant aux valeurs, se réfère également à des questions métaphysiques, lesquelles peuvent constituer autant d'arguments pour des pouvoirs totalitaires afin, sous couvert d'idéologie, de s'immiscer dans les moindres actions des gens. Telle a été ou est encore la situation qui prévaut dans les pays communistes. Savoir si autrui enfreint votre action, voilà bien une question que l'on peut trancher empiriquement sans s'aventurer dans le domaine de la métaphysique. Il faut préciser que Yin Haiguang lui-même avait été influencé par l'empirisme logique, lequel avait progressivement nourri son aversion pour la métaphysique. Pour toutes ces raisons, on peut dire que les polémiques entre néo-confucéens et libéraux taiwanais sur la question de la liberté reflètent aussi les divergences entre l'idéalisme allemand d'une part et la tradition empirique anglaise d'autre part.

\section{La distinction de Berlin sur les deux types de liberté et le contexte de la guerre froide}

La distinction de Berlin entre libertés positive et négative a exercé une influence profonde sur la pensée politique occidentale au $\mathrm{Xx}^{\mathrm{e}}$ siècle. L'essai sur les deux concepts de liberté remonte à un discours prononcé le 10 janvier 1958

11. Isaiah Berlin, Four Essays on Liberty, Oxford, Oxford University Press, 1969, p. 122.

12. Ibid., p.131sq. 
à Oxford ${ }^{13}$. Cependant, il me faut insister sur un point auquel même le monde académique chinois n'a que peu prêté attention: Zhang Foquan, dans son livre de 1953 intitulé Liberté et droits de l'homme, évoquait déjà une distinction très proche qu'il associait à l'époque aussi à la question de la liberté formelle (xingshi ziyou) ou réelle (shizhi ziyou) ${ }^{14}$. Ce livre, paru cinq années avant le discours de Berlin, s'opposait à la «liberté positive» avec des arguments que n'aurait pas désavoués le philosophe anglais.

Le moment où Berlin propose sa distinction entre les deux types de liberté correspond à une phase très tendue de la guerre froide ${ }^{15}$. À l'époque, tout un groupe d'intellectuels libéraux occidentaux réfléchissait aux causes de la montée du nazisme et du fascisme d'avant guerre et, plus généralement, aux sources de la pensée totalitaire avec pour préoccupation la lutte contre la menace communiste. En dehors d'I. Berlin, on comptait parmi eux Jacob Leib Talmon (1916-1980), Friedrich A. von Hayek (1899-1992) ou encore Karl Popper (1902-1994). À la distinction de Berlin faisaient écho deux autres : celle de Talmon, entre « démocratie totalitaire » (jiquan minzhu) et «démocratie libérale» (ziyou minzhu) ${ }^{16}$; et celle de Hayek qui évoquait «la tradition française » et la «tradition anglaise ${ }^{17}$. Quant à Popper, il se situait lui aussi sur une ligne très proche dans des livres comme La Pauvreté de l'historicisme (1957) ou La Société ouverte et ses ennemis (1945) quand il recherchait les sources originelles du totalitarisme au cœur de la pensée de Platon, Hegel ou Marx. À partir de 1949, Taiwan entra à son tour dans ce contexte politique général de guerre froide. Dès lors, les libéraux comme Yin Haiguang ou Zhang Foquan se retrouvèrent peu ou prou dans une configuration semblable à celle de leurs pairs occidentaux, et leur pensée allait donc suivre une ligne très comparable.

En Occident, les thèses de Berlin suscitèrent bien des discussions. À partir des années 1980, elles rencontrèrent deux types de critiques. Les premières émanèrent

13. Voir Michael Ignatieff, Isaiah Berlin: A Life, New York, Metropolitan Books, 1998, p. $225 s q$.

14. Foquan Zhang, Ziyou yu renquan (Liberté et droits de l'Homme), Taibei, Taiwan Shangwu Yinshuguan chongyin, 1993. Voir notamment le chapitre 6.

15. Quentin Skinner indique à propos de Berlin: «Le texte de Berlin est fortement marqué par les divisions géopolitiques et idéologiques des années 1950 ». Quentin Skinner, «A Third Concept of Liberty», Proceedings of the British Academy, vol. 117 (2002), p. 264.

16. Talmon Jacob Lei, The Origins of Totalitarian Democracy, Londres, Secker \& Warburg, 1952; Political Messianism: The Romantic Phase, Londres, Secker \& Warburg, 1960.

17. Friedrich A. Hayek (von), The Constitution of Liberty, Londres, Routledge \& Kegan Paul, 1960, p. 54-56. 
des républicains (gonghezhuyi), représentés notamment par des intellectuels comme Quentin Skinner (1940-) ou Philip Pettit (1945-). Les secondes provenaient des communautariens avec, au premier chef, la figure de Charles Taylor (1931-). Les républicains, qui reconnaissaient certes l'intérêt de la notion de liberté négative, la déclinèrent cependant sur deux modes, libéral et républicain. Ils firent dès lors de cette «liberté négative républicaine» un «troisième concept de liberté». La différence entre une telle conception et celle des libéraux tient aux points suivants: pour les libéraux, la liberté négative s'ancre dans les droits de l'individu et considère l'État comme un «mal nécessaire». Dès lors, il n'est de «liberté de l'État» (guojia ziyou) dont on puisse parler. Au contraire, pour les républicains, la liberté individuelle ne peut être préservée que dans le cadre d'une communauté naturelle autonome. Pour cette raison, il n'est pas possible de différencier liberté de l'individu et liberté de l'État. Plus encore, pour protéger la «liberté de l'État», des « vertus civiques» (gongmin de dexing) sont nécessaires ${ }^{18}$.

L'exemple de Charles Taylor et de son article intitulé What's Wrong with Negative Liberty? permettra de se faire une idée du jugement que les communautariens portent sur le concept de liberté négative d'I. Berlin ${ }^{19}$. Pour Taylor, ce dernier, simple «élimination d'obstacles extérieurs», n'est qu'un «concept d'opportunité » (jihui gainian, opportunity-concept). Une telle liberté ne porte que sur ce que l'on peut faire, ou, en d'autres termes, sur l'espace dont on dispose pour effectuer des choix et agir comme on l'entend. Elle ne s'intéresse pas au fait de savoir si nous sommes réellement à même d'effectuer de tels choix. Taylor souligne que l'on ne peut, à partir de ce concept de liberté, prendre en compte l'un des objectifs centraux du libéralisme, à savoir la réalisation de l'individu (geren de ziwo shixian) ${ }^{20}$. Si l'on aborde ce dernier problème, la liberté

18. Sur le concept de liberté dans le républicanisme, voir les textes suivants: (1) Quentin Skinner, «The idea of Negative Liberty: Philosophical and Historical Perspectives», in Rorty Richard et al. (éd.), Philosophy in History: Essays on the Historiography of Philosophy, Cambridge, Cambridge University Press, 1984, p. 193-221; (2) Quentin Skinner, «The Paradoxes of Political Theory », The Tanner Lectures on Human Values, VII (1986), p. 225-250; (3) Quentin Skinner, «A Third Concept of Liberty», op. cit., p. 237-268; (4) Philip Pettit, «Negative Liberty, Liberal and Republican», European Journal of Philosophy, vol. 1 (1993), p. 15-38; (5) Philip Pettit, «A Definition of Negative Liberty», Ratio, New Series, vol. 2 (1989), p. 153-168.

19. Cet article figure dans: Philosophy and the Human Sciences : Philosophical Papers 2, Cambridge, Cambridge University Press, 1985, p. 211-229.

20. Rappelons que le libéralisme classique pose que chaque personne est libre de se réaliser comme elle l'entend. La responsabilité de l'État est simplement de lui fournir un cadre dans lequel elle peut librement poursuivre les valeurs qu'elle souhaite. On retrouve là la conception dite «négative» de la liberté. Mais Lee Ming-huei souligne donc que pour Taylor, la question, au-delà des principes, est de savoir comment quelqu'un peut acquérir la capacité pratique de faire des choix qui lui permettront de se réaliser. N.d.T. 
ne peut plus se limiter à constituer un simple «concept d'opportunité», mais elle doit devenir un «concept applicable» (yunzuo gainian, exercise concept). Dès lors, la liberté de l'individu dépend aussi de sa capacité pratique à agir de façon autonome (zizuo zhuzai) et à ainsi déterminer les orientations de sa vie. Analysant les mobiles de nos actions (ou nos objectifs), nous ne pouvons pas ne pas distinguer ceux qui sont réels et importants de ceux qui sont artificiels et mineurs. En d'autres termes, nous devons reconnaître que le choix de nos valeurs [manifesté à travers certains mobiles ou objectifs que nous identifions comme importants] n'est pas une question simplement subjective, mais dépend aussi d'éléments objectifs. De façon générale, la question de la réalisation de soi une fois abordée, on est obligé de prendre en compte l'idée de liberté positive et de penser plus largement la liberté comme «une capacité de réaliser nos buts ». Cependant, pour Berlin, le problème réside dans le fait qu'une fois reconnue l'existence d'une dimension objective dans nos choix de valeurs, il est bien possible que l'État ou la société, s'auto-investissant de la mission d'aider l'individu à réaliser ce qu'il y a de réel et d'important, ne s'immiscent dans la moindre de ses actions pour ainsi exercer sur lui une pression collective. C'est contre cela que Berlin s'érige, soucieux de préserver la liberté négative, «ligne Maginot» contre toutes les velléités d'oppression. Mais pour Taylor, une telle ligne ne protège rien. Plus encore, il avance que les jugements objectifs dans le domaine des choix de valeurs ne mènent pas fatalement au totalitarisme. Autrement dit, il n'y a pour lui aucune relation logique nécessaire entre la liberté positive et le totalitarisme.

\section{La critique des communautariens à l'égard des libéraux}

Si l'on considère les deux grands problèmes qui opposaient libéraux taiwanais et nouveaux confucéens, les idées de ces derniers recoupent largement celles des communautariens. Pour préparer le lecteur à nos développements ultérieurs, nous devons au préalable faire retour sur le terreau intellectuel du communautarisme.

Le communautarisme est un courant de pensée politique anglo-saxon apparu dans les années 1980. Hormis Charles Taylor, il a pour représentants majeurs Michael J. Sandel (1953-), Alasdair MacIntyre (1929-) ou encore Michael Walzer (1937-). Issu d'une critique de l'œuvre maîtresse du philosophe contemporain libéral John Rawls (1921-2002), Théorie de la justice, ce courant s'est ensuite développé en critique complète de tous les présupposés fondamentaux du libéralisme.

Une différence fondamentale sépare les libéraux classiques de John Rawls. Les premiers, à l'instar de Jeremy Bentham ou de John Stuart Mill, tiraient leurs 
bases théoriques de l'utilitarisme (gonglizhuyi), alors que Rawls pour sa part allait partir de l'éthique (déontologique) kantienne. Il estimait en effet que toute éthique téléologique ${ }^{21}$ - et cela englobait alors l'utilitarisme - ne pouvait suffire à instituer le principe d'une «justice comme équité» (zhengyi ji gongpin, justice as fairness). Chez les communautariens, Michael Sandel allait critiquer avec vigueur cette éthique déontologique et l'idée qui lui était associée "d'une priorité du juste sur le bien» (Zhengque youxian yu shan, the priority of the right over the good) ${ }^{22}$. Dans Après la vertu (1981), Alasdair MacIntyre allait également pourfendre «l'éthique kantienne du devoir» et l'utilitarisme de Mill, jugeant qu'ils devaient tous deux endosser la responsabilité de la détresse morale dans laquelle avait sombré la société occidentale moderne. Pour la surmonter, il appelait alors de ses vœux un retour à Aristote et à une tradition éthique valorisant «le caractère moral (dexing) et l'action morale (dexing)». Taylor allait quant à lui chercher son inspiration dans le concept de Hegel de «vie éthique» (lunli, Sittlichkeit), et retourner alors aussi indirectement à la tradition d'Aristote.

En dehors de leurs différences sur le plan éthique, un autre clivage fondamental divise les communautariens et les néo-libéraux représentés notamment par Rawls, clivage qui touche à la relation entre l'individu et la communauté. Sandel, MacIntyre et Taylor sont en effet critiques à l'égard du concept du sujet (ou du «Moi», ziwoguan) présupposé par les libéraux. Dans Le Libéralisme et les limites de la justice, Sandel critique «le sujet individué de façon préalable ${ }^{23}$ » de Rawls ou le concept de « unencumbered self ${ }^{24}$ », qui fait référence à un Moi abstrait et détaché de toute relation. Dans Après la vertu, MacIntyre fait remonter la vision du «Moi» des libéraux, qu'il critique, à l'émotivisme moral ${ }^{25}$ des Lumières. Enfin, dans Les Sources du moi (1989) Charles Taylor s'en prend à «l'atomisme ${ }^{26}$ du Moi présupposé par les libéraux.

21. Une éthique téléologique fait dériver le devoir ou l'obligation morale d'une fin considérée comme bonne ou désirable. L'utilitarisme en est un bon exemple. Elle s'oppose à l'éthique déontologique centrée non d'abord sur le résultat de l'action, mais sur le caractère moral de l'intention. N.d.T.

22. Sur la critique de Sandel de la Théorie de la justice de Rawls, voir son ouvrage Liberalism and the Limits of Justice, Cambridge, Cambridge University Press, 1982 et 1998 (seconde édition).

23. xianxing getihua de zhuti, antecedently individuated subject.

24. wu qianlei de ziwo. Voir Sandel Michael, «The Procedural Republic and the Unencumbered Self», Political Theory, vol. 12, 1984, p. 81-96; et Reich Robert B. (éd.), The Power of Public Ideas, Cambridge (États-Unis), Harvard University Press, 1988, p. 109-121.

25. qingxuzhuyi daodeguan.

26. yuanzilun. 
Sandel souligne que la conception déontologique (théorie des devoirs) adoptée par Rawls se réfère, sur un plan logique, à un «sujet individué de façon préalable» (antecedently individuated subject). Afin d'expliquer ce point, il se livre dans l'introduction au Libéralisme et les limites de la justice ( $1^{\mathrm{re}}$ section) à une analyse minutieuse de l'influence de l'éthique de Kant et de sa conception du Moi sur la Théorie de la justice de Rawls. Cette influence est d'abord manifeste dans la position que Rawls adopte sur la théorie des devoirs (yiwulun) et Sandel n'hésitera dès lors pas à qualifier sa pensée de «libéralisme déontologique » ${ }^{27}$, qu'il évoquera de la manière suivante:

Le «libéralisme déontologique» est avant tout une théorie sur la justice, et en particulier sur la priorité à accorder à la justice parmi les idéaux politiques ou moraux. Sa thèse centrale peut être énoncée de la manière suivante: la société, composée d'une pluralité de personnes avec chacune ses propres objectifs, intérêts ou conceptions du bien, est mieux gérée quand elle est gouvernée par des principes qui ne présupposent pas eux-mêmes quelque conception particulière du bien. Ce qui justifie avant tout ces principes et leur rôle régulateur n'est pas qu'ils optimisent le bien-être social ou qu'ils promeuvent le bien, mais plutôt qu' ils se conforment au principe du juste, une catégorie morale donnée préalablement à celle de bien et qui lui est indépendante ${ }^{28}$.

Nul n'ignore que dans l'éthique de Kant, le socle du devoir ou des valeurs morales repose avant tout et ultimement sur les principes auxquels se tient le sujet (c'est-à-dire sur ses intentions) et non pas sur les buts visés par l'action morale ou sur quelque résultat escompté. Il s'agit là d'une conception classique de l'éthique déontologique. Sandel insiste bien sur ce pointen indiquant qu'une telle éthique affirme la priorité du sujet sur l'objet (du Moi sur le but) et que le sujet ainsi envisagé assied alors le caractère nécessaire et universel des principes moraux. Dès lors, pour une telle éthique, les principes moraux ont également la priorité sur le bien (daode faze youxian yu shan). Dans La Critique de la raison pratique, Kant est limpide sur ce point: «Le concept du bien (Guten, shan) et du mal (Bösen, e) ne doit pas être déterminé avant la loi morale (à laquelle, d'après l'apparence, il devrait servir de fondement), mais seulement $[\ldots]$ après cette loi et par elle ${ }^{29}$.»

27. yiwulun ziyouzhuyi, deontological liberalism.

28. Michael Sandel, Liberalism and the Limits of Justice, op. cit., seconde édition, p. 1.

29. «[...] da $\beta$ nämlich der Begriff des Guten und Bösen nicht vor dem moralischen Gesetze (dem er dem Anschein nach sogar zum Grunde gelegt werden müßte), sondern nur [...] nach demselben und durch dasselbe bestimmt werden müsse», Kritik der praktischen Vernunft (Critique de la raison pratique), in Kants Gesammelte Schriften, Akademieausgabe, vol. 5, p. 62 sq. Pour cette citation de Kant, nous utilisons la traduction que donne François Picavet de La Critique de la raison pratique, Paris, PUF, «Quadrige», 2000, p. 65. N.d.T. 
Sandel indique égalementque le sujet présupposé par l'éthique déontologique kantienne est un sujet transcendantal, qu'il se situe donc hors du champ de l'expérience et n'est pas limité par la loi de causalité (yinguolï). À partir d'un ensemble de concepts tels que le phénomène ou la chose en soi, le monde sensible ou intelligible, Kant donne une sorte d'ancrage métaphysique à son sujet transcendantal. Pour Rawls, le sujet transcendantal de Kant et la structure métaphysique que celui-ci institue a priori manquent de contenu empirique et il en vient donc à les critiquer en dénonçant leur caractère obscur (hanhun) et arbitraire (wuduan). Il va dès lors s'efforcer de séparer la structure fondamentale de l'éthique kantienne de son présupposé (yushe) métaphysique, pour la reconstruire à partir «des canons d'un empirisme raisonnable ${ }^{30}$ ». Sandel, dès lors, qualifiera de «déontologie à visage humien» cette éthique reconstruite par Rawls.

Pour reconstruire l'éthique kantienne et remplacer la supposition (yushe) métaphysique d'un «royaume des fins» (Reich der Zwecke, mudi wangguo), Rawls a avancé l'hypothèse théorique de la «position originelle» (original position, yuanchu zhuangtai). Pour dire les choses simplement, cette position originelle est une sorte de théorie du contrat: en vertu de cette hypothèse, chaque individu est égal et, du fait d'un "voile d'ignorance» (veil of ignorance, wu zhi zhi mou), il ne sait rien de sa position sociale, de sa race, de son sexe, de sa classe, de sa richesse, de ses talents, de sa force ou de toute autre qualité qui lui aurait été naturellement impartie. Dès lors, en déterminant le contrat qui les lie, les individus n'ont plus à prendre en compte les circonstances sociales ou naturelles particulières et sont dès lors véritablement à même d'atteindre l'idée de ce qu'est «la justice comme équité» (zhengyi ji gongpin). Ainsi, Rawls espérait préserver l'esprit fondamental de l'éthique kantienne tout en évitant les critiques adressées à sa supposition métaphysique.

Néanmoins, d'après Sandel, dès lors que Rawls continue, à partir d'une théorie des devoirs, à affirmer «la priorité du juste sur le bien» (zhengque youxian yu shan) et celle «du Moi sur les fins» (ziwo youxian yu mudi) ${ }^{31}$, son concept du Moi ne peut éviter de tomber sous le coup du même type de critiques que celles qui furent adressées à Kant. Les critiques de Sandel à l'endroit de Rawls reposent sur trois grands points:

30. heli de jingnianlun zhi guizhun. Voir John Rawls, «The Basic Structure as Subject», American Philosophical Quaterly, vol. 14, 1977, p. 165.

31. Sandel note que Rawls évoque «a subject of possession, individuated in advance and given prior to its ends » (p. 59) ou «a self regarded as given prior to its ends » (p. 57). Michael J. Sandel, Liberalism and the Limits of Justice, Cambridge, Cambridge University Press, 1998. 
- La conception du Moi de Rawls serait tout d'abord partiale (pianmian de) en ce qu'elle se focaliserait juste sur la capacité de choisir un but ou un objet. Elle prendrait alors la forme d'une activité de notre volonté, au détriment d'autres types d'activités comme nos activités cognitives ${ }^{32}$.

- Cette conception du Moi serait également superficielle, parce que le sujet tel que Rawls l'entend est déjà individué (getihua de) avant même tout choix de but ou d'objet. L'acte même de choisir n'aurait donc pas d'impact sur son identité ${ }^{33}$. Dès lors, Rawls écarte même la possibilité que l'engagement du sujet (par exemple à l'égard de la communauté) puisse avoir quelque impact sur son identité ${ }^{34}$.

- La perspective de Rawls sur la communauté politique (zhengzhi shiqun) serait enfin tout simplement pauvre car, d'une part, participer à une telle communauté n'est pour lui que l'un des objectifs, parmi d'autres, que peut se donner un sujet préalablement individué (antecedently individuated subject); d'autre part, une telle participation ne peut avoir lieu que dans une société libre (ziyou shehui) telle que définie par sa conception de la justice. Partant, le sens de la communauté (shiqun yishi, sense of community) pour Rawls n'est que l'attribut d'une «société bien ordonnée» (well-ordered society), non pas son élément constitutif $^{35}$.

Si nous résumons ce qui a été énoncé auparavant, la critique de Sandel à l'égard de la conception de la personne de Rawls se ramène aux éléments suivants : Rawls n'aurait pas prêté assez d'attention au fait que nos aspirations en vue de certaines fins, ou la confiance dont nous sommes investis en vue de les atteindre, sont des éléments constitutifs de notre identité en tant que personnes (ziwo rentong); il n'aurait pas non plus considéré le fait que le sens de la communauté est un élément constitutif d'une communauté politique. Tout ceci est en définitive lié à sa position en matière de théorie des devoirs, au subjectivisme moral $^{36}$ (daode zhutizhuyi) qui s'y associe et à sa conception d'une «priorité du juste sur le bien ».

32. Michael Sandel, Liberalism and the Limits of Justice, op. cit., p. 56-59.

33. Sandel reproche à Rawls de soustraire le sujet à toute expérience en fixant son identité préalablement une fois pour toutes. Ibid., p. 62.

34. Ibid., p. 61-62.

35. Ibid., p. 64. «As a person's values and ends are always attributes and never constituents of the self, so a sense of community is only an attribute and never a constituent of a well-ordered society». N.d.T.

36. Sur l'emploi du terme «subjectivisme moral» pour décrire le concept du Moi de Rawls, voir Stephen Mulhall et Adam Swift, Liberals and Communitarians, Oxford, Blackwell, 1992, p. 55-59. 


\section{Les confucéens contemporains, entre libéraux et communautariens}

Ces rappels préliminaires sur la philosophie politique occidentale peuvent nous aider à mieux comprendre la position des confucéens contemporains et, plus largement, du confucianisme traditionnel ${ }^{37}$. Nous pensons en effet que tant les confucéens contemporains que le confucianisme traditionnel incarné par des figures comme Confucius ou Mencius, se situent entre libéralisme et communautarisme. Il n'est pas difficile de voir que les communautariens et les néo-confucéens se retrouvent sur trois points: (1) la conception des deux types de liberté; (2) les conceptions de l'individu et de la communauté; (3) la conception de la tradition.

Non seulement les néo-confucéens ne nient pas l'importance de la liberté négative, mais ils estiment au contraire que cette dernière est un préalable à une politique démocratique et à la pensée des droits de l'homme. Ils reconnaissent également que l'une des raisons essentielles pour laquelle la Chine n'a pas été à même, dans le passé, d'instituer un système démocratique, tient au fait qu'une conception de la «liberté négative» lui faisait défaut. Sur ce point, les confucéens contemporains rejoignent les libéraux. Cependant, ils vont plus loin en insistant sur un point: théoriquement, la liberté négative doit être un préalable à la liberté positive; pratiquement cependant, évoquer simplement la liberté négative ne suffit pas pour s'opposer au totalitarisme. Sur ce point précisément, ce sont les communautariens que rejoignent les confucéens contemporains. Le confucianisme classique ne possède pas une telle idée de la liberté négative et la liberté morale qui lui est centrale ${ }^{38}$ se rapproche sans aucun doute de la liberté positive.

Au sujet des liens entre l'individu, la communauté et la tradition, les connivences entre confucéens contemporains et communautariens sont encore plus évidentes. En 1956, Zhang Qiyun, qui était alors ministre de l'éducation de la République de Chine (Taiwan), avait critiqué le libéralisme dans un article intitulé «les trois vérités d'une politique démocratique». Yin Haiguang lui avait alors répondu dans les colonnes de Chine libre avec un texte qui avait pour titre «La conception de la démocratie du ministre de l'éducation Zhang Qiyun: une démocratie monarchique». Avait alors suivi une série d'attaques du Kuomintang contre les libéraux. Au même moment, Xu Fuguan publiait successivement deux articles dans Tribune démocratique et dans le Quotidien des chinois d'outre-mer

37. Lee Ming-huei fait allusion ici aux grands textes confucéens de l'antiquité chinoise, notamment aux quatre livres. N.d.T.

38. Il s'agit naturellement ici d'une interprétation de l'auteur de la pensée confucéenne classique, laquelle n'a pas été conceptualisée autour de la notion de liberté. N.d.T. 
(Huaqiao ribao) de Hong Kong dans lesquels il soutenait le libéralisme et prenait la défense de Yin Haiguang. Dans le premier de ces deux textes, il expliquait que «même si une personne n'embrasse pas le libéralisme ou ne peut simplement se résigner à devenir libérale, elle devra, dans le processus de développement de ses connaissances et de sa personnalité, passer par le libéralisme ${ }^{39}$. Après avoir réaffirmé la valeur du libéralisme, il insistait sur les points suivants:

Les libéraux se sont libérés de la tradition et de la société, ce qui ne revient pas à dire qu'ils les ont simplement niées. Ils en ont fait une réévaluation, [...] afin de leur donner de nouveaux contenus et ainsi, ils les ont remodelées de façon plus riche et plus appropriée. Les libéraux vont continuer de vivre dans le courant de la tradition et de la société. Une telle vie n'est néanmoins pas passive ou négative; bien au contraire, elle est active, positive, et offre une impulsion qui ne cesse de recréer la société et la tradition. De la sorte, ces dernières ne constituent plus des forces aveugles, mais, innervant nos facultés rationnelles et morales, elles en deviennent progressivement aussi le produit. Dès lors, le libéralisme ne revient plus simplement à libérer l'esprit et constituer l'individu car ce faisant, il façonne aussi la communauté. Certes, il est des libéraux qui ne reconnaissent que l'individu et non la communauté. Néanmoins, l'histoire nous montre que la vitalité de l'individu modèle nécessairement aussi celle de la communauté. Par conséquent les pays libéraux sont dans l'ensemble, historiquement, les plus puissants et ceux qui progressent le plus ${ }^{40}$.

Ainsi le «libéralisme» tel que Xu Fuguan l'entend et l'affirme n'est pas celui que critiquent les communautariens (c'est-à-dire le libéralisme de ceux qui «ne reconnaissent que l'individu et non la communauté»). Bien au contraire, il se rapproche des conceptions communautariennes puisque l'individu dont il est question n'est pas compris d'abord comme isolé, mais comme modelé par la communauté et par la tradition. Il est évident qu'il hérite là de conceptions confucéennes traditionnelles sur l'individu et la société. Comme chacun le sait, les confucéens traditionnels mettaient fortement l'accent sur la communauté et les relations interpersonnelles. En témoigne la réaction spontanée de Confucius, relatée dans ses Entretiens (18:6), quand, [quelque peu frustré], il commente à son disciple Zilu dans les termes suivants les boutades dont ils venaient tous deux de faire l'objet de la part des ermites Zhang Ju et Jie Ni: «Je ne peux m'associer aux oiseaux et aux bêtes; si je ne le fais avec des gens comme

39. Xu Fuguan, Rujia zhengzhi sixiang yu minzhu ziyou renquan (Pensée politique confucéenne et droits de l'homme et liberté en démocratie; textes de Xu Fuguan rassemblés et édités par Xiao Xinyi), Taibei, Taiwan xuesheng shuju, 1988, p. 289.

40. Xu Fuguan, Xueshu yu zhengzhi zhijian (Entre travail académique et politique), Taibei, Taiwan xuesheng shuju, 1980, p. 459. 
eux, avec qui le pourrais-je ${ }^{41}$ ?» Mais une telle position n'empêche néanmoins pas Confucius de reconnaître, [pour employer un vocabulaire contemporain], l'indépendance de la personne. Aussi nous dit-il dans un autre passage de ces mêmes Entretiens que : «Des armées peuvent être privées de leur général, mais il n'est d'homme, même ordinaire, que l'on puisse priver de sa volonté» $(9: 26)$. Pour ce qui est des relations entre l'individu et la société, Confucius n'est pas l'apôtre de quelque «collectivisme» (jitizhuyi) que ce soit, mais il ne se serait pas non plus senti proche de l'individualisme occidental moderne. Il affirme à la fois l'indépendance de l'individu et l'existence de liens étroits entre lui et la société. En d'autres termes, si l'individu appartient intrinsèquement selon lui à la société, il la dépasse néanmoins également. Pour différencier cette position de celle de l'individualisme (gerenzhuyi) occidental moderne, William Theodore de Bary préfère parler de «personnalisme» (rengezhuyi) $)^{42}$. Taylor estime lui aussi que «Le moi n'est tel que parmi les autres moi, on ne peut jamais l'évoquer sans référence aux autres moi qui l'entourent ${ }^{43}{ } »$. À partir de tous les points que nous venons d'évoquer, on comprend déjà les affinités entre confucéens, tant contemporains que classiques, et le communautarisme.

À partir des explications de Xu Fuguan évoquées auparavant, on voit également que les confucéens contemporains, quand ils mettent l'accent sur le lien entre la communauté et l'individu, insistent aussi sur ce qui relie ce dernier à la tradition. L'une des divergences majeures entre les néo-confucéens et les libéraux taiwanais tient précisément à leur appréciation différente de la tradition. Yin Haiguang, dans son texte sur «la valeur de la tradition», exprimait sa position dans les termes suivants :

Ce que l'on appelle tradition, c'est simplement un instrument auquel se réfère la communauté pour subsister. La tradition n'est pas la tablette ancestrale d'un lignage [devant laquelle on s'incline avec révérence], elle n'est pas non plus un objet d'adoration ou un Boddhisatva auquel on fait des offrandes. Savoir si on peut la rectifier, la

41. Dans ce passage des Entretiens (18: 6), Confucius, en route avec son disciple Zilu, envoie ce dernier demander à deux ermites travaillant dans un champ où se trouve le gué qui leur permettra de poursuivre leur route. Les ermites invitent Zilu à suivre ceux qui «évitent le monde » (bishi, c'est-à-dire ceux qui, comme eux, mènent une vie d'ermite) plutôt que celui qui évite, de manière sélective, certains types de personnes (ils visent alors Confucius) N.d.T.

42. W. Theodore de Bary, «Individualism and Personhood», in W. Theodore de Bary (éd.), Asian Values and Human Rights : A Confucian Communitarian Perspective, Cambridge (États-Unis), Harvard University Press, 1988, p. 25.

43. «One is a self among other selves. A self can never be described without references to those who surround it»: Charles Taylor, Sources of the Self, Cambridge (États-Unis), Harvard University Press, 1989, p. 35. 
conserver ou la modifier, tout cela dépend complètement de sa pertinence pour notre vie. Ce critère une fois fixé, toute attitude vis-à-vis d'elle (rectification, conservation, modification) est possible car on ne s'embourbera alors pas dedans ${ }^{44}$.

Nous sommes là devant une «théorie instrumentale de la tradition », cette dernière étant pensée comme une ressource au service d'une communauté. Sa capacité à offrir un instrument adapté aux nécessités de la vie détermine alors sa valeur. Si l'on comprend ainsi la tradition, sa relation aux hommes n'a rien d'intrinsèquement essentiel.

Le point de vue de Xu Fuguan sur la tradition est complètement différent. Il avait autrefois traduit un texte du philosophe japonais Mutai Risaku ${ }^{45}$ intitulé Le problème de la tradition dans la philosophie de l'histoire ${ }^{46}$ et il était parti de là pour écrire un discours dont le titre était À propos de la tradition (lun chuantong $)^{47}$. Il y opérait une distinction entre basse (diciyuan de chuantong) et haute (gaociyuan de chuantong) traditions. Les coutumes et habitudes rentraient dans le champ de la première, laquelle se manifestait dans les choses les plus concrètes, possédait un caractère assez statique et n'était pas vécue comme telle, de façon très consciente ${ }^{48}$. Quant à la haute tradition, Xu Fuguan l'évoquait dans les termes suivants :

La haute tradition, c'est la découverte, à partir des choses les plus concrètes de la basse tradition, qu'il existe en filigrane, innervant celle-ci, des fins et un esprit plus originels. La haute tradition est souvent modelée par les fondateurs de religion, les Saints, artistes majeurs, grands esprits, etc., d'une nation. Elle appartient au spirituel, est intangible et ne peut être réappropriée que par un retour sur soi ou à travers un processus de prise de conscience. Une telle réappropriation ouvre aussi la possibilité d'une critique de la basse tradition. Cet examen critique est naturellement l'occasion de relier les différentes dimensions du temps (passé, présent, futur) les unes

44. Lin Zhenghong (éd.), Yin Haiguang Quanti (Euvres complètes de Yin Haiguang), Taibei, Guigua tushu gongsi, 1990, vol. 13 (Xueshu yu sixiang), p. 281.

45. Mutai Risaku (1890-1974). Ce philosophe japonais fut un élève de Nishida Kitarô à l'université de Kyoto, qui travailla aussi en Allemagne sous la direction de Husserl. Spécialiste de la philosophie allemande, ses intérêts le portèrent néanmoins vers de nombreux autres domaines. C'est lui qui a présenté au Japon la thèse de Hans Naumann sur la culture. N.d.T., à partir de précisions de Naoko Hirata.

46. Ce texte a été intégré dans Wutai Lizuo (Mutai Risaku), Lunzhan yu shishu, Taibei, Zhiwen chubanshe, 1982, p. 190-200.

47. Ce texte figure dans Xu Fuguan, Хи Fuguan wenlu xuancui (sélection d'essais de Xu Fuguan éditée par Xiao Xinyi), Taibei, Taiwan xuesheng shuju, 1980, p. 105-106. Ces explications relatives à la tradition sont également citées dans un autre texte du même recueil intitulé «Chuantong yu wenhua» (Tradition et culture).

48. Ibid., p. 110. 
aux autres et de réfléchir à leurs articulations. [...] Dès lors, en elle-même, la haute tradition porte l'idée d'un dépassement du traditionnel (chao chuantongxing). Plus concrètement, elle possède les caractéristiques suivantes: (1) elle est idéaliste (de la même façon que l'amour universel chrétien est idéaliste et distinct des formes rituelles particulières de la basse tradition). (2) Comme on se la réapproprie au terme d'un processus de retour sur soi et de prise de conscience, elle porte nécessairement une dimension critique. (3) Parce qu'elle est critique, elle est aussi dynamique. (4) Étant dynamique, elle est en constante reconfiguration, héritant du passé et le dépassant à la fois ${ }^{49}$.

En comparant les conceptions de Yin Haiguang et de Xu Fuguan de la tradition, il n'est pas difficile de distinguer deux points de divergence majeurs. Tout d'abord, la «tradition» telle qu'elle est prise en compte par Yin est une réalité objective dont on n'a pas nécessairement conscience. Comme telle, elle s'apparente à la «basse tradition». Pour Xu Fuguan cependant, seule la haute tradition véhicule l'essence d'une tradition. Elle n'est pas un instrument utilisable en fonction de tel ou tel dessein, mais elle représente la conscience spirituelle d'une nation ${ }^{50}$ et est alors porteuse d'idéaux. En second lieu, la tradition existe pour Yin Haiguang de façon statique et il ne tient pas compte de sa dimension historique ou de sa vie propre. Pour $\mathrm{Xu}$, elle est au contraire dynamique, vivante, elle se perpétue et se dépasse elle-même. Pensée comme un instrument par Yin, elle est subjectivée par $\mathrm{Xu}$, car seul un sujet est à même de prendre conscience de lui-même.

Les confucéens contemporains héritent clairement, sur ces thèmes, de conceptions bien connues du confucianisme classique. Ainsi, le rite $(l i)$ tant valorisé est-il un élément de la tradition. Confucius lui-même se considérait comme l'héritier de la culture de Zhou. Il avait «transmis les doctrines de Yao et de Shun, comme s'ils avaient été ses ancêtres et comme s'il s'était référé aux règles de Wen et de Wu en les prenant pour modèle» (Zhongyong, 30); il se caractérisait aussi comme transmetteur, plus que créateur, ayant foi dans le passé et le chérissant (Entretiens, 7.1). Mencius considérait quant à lui qu'il perpétuait les trois générations de sages rois ${ }^{51}$ et la Voie de Confucius. Pour ce dernier cependant, la tradition pouvait être adaptée aux besoins de l'époque. Aussi précisait-il que «la dynastie des Yin (les Shang) a hérité des rites des Xia et l'on sait ce qu'elle a ajouté ou retranché; la dynastie des Zhou a hérité des rites des Yin et l'on sait ce qu'elle a rajouté ou retranché; d'autres héritent des

49. Ibid., p. 110-111.

50. Le terme de minzu, est toujours délicat à traduire et le mot de nation ne rend que très imparfaitement son sens. Il se rapproche ici des idées de culture et de civilisation. N.d.T.

51. Les fondateurs de dynastie: Yu le Grand (sous les Xia), Tang (sous les Shang) et Wen et $\mathrm{Wu}$ (sous les Zhou). N.d.T. 
Zhou et la postérité le saura aussi » (Entretiens 2 :23). Ce n'est pas là considérer la tradition comme un instrument mais reconnaître, à l'instar de Xu Fuguan, qu'elle se «perpétue et se dépasse elle-même». Chez les communautariens, MacIntyre reproche particulièrement à l'individualisme occidental moderne (qu'il considère comme étant au fondement du libéralisme) de ne pas comprendre la signification de la tradition dont nous sommes tous dépositaires et ainsi d'opposer tradition et raison ${ }^{52}$. Nous voyons là d'évidentes affinités entre confucéens et communautariens.

Ce que nous venons d'évoquer n'est cependant qu'un aspect de la question. Entre les confucéens et le libéralisme d'un Rawls et de ses pairs, il existe en effet aussi des points de rapprochement. Il ne fait nul doute qu'au sein du courant principal du confucianisme classique, incarné par des figures comme Confucius ou Mencius, on trouve aussi une éthique des devoirs. Cela est manifeste chez Mencius dans sa «différenciation entre le sens du juste et l'intérêt» (yi li zhi bian) ou quand il évoque «la dimension intérieure du sens de l'humain et du sens du juste» (ren yi nei zai). À l'instar de l'éthique déontologique kantienne qui affirme «la priorité de la loi morale sur le bien » ou du libéralisme déontologique de Rawls pour lequel «le juste a priorité sur le bien», Mencius lui aussi insiste sur le fait que «le sens du juste vient avant toute considération d'intérêt» (xian yi hou li). La discussion sur «le sens du juste et l'intérêt» inaugure son ouvrage à travers un dialogue qui met en scène Mencius face au roi Hui de Liang ${ }^{53}$. Sa signification est la suivante: le «juste» et «l'intérêt» sont deux modalités du «bien» essentiellement différentes et non équivalentes. Quand elles rentrent en conflit, le «juste» doit l'emporter sur «l'intérêt». On retrouve une idée proche chez le penseur américain John R. Silber qui, dans son interprétation de l'éthique de Kant, évoque une "hétérogénéité du bien» (the heterogeneity of the good, shan zhi yizhixing ${ }^{54}$. Reconnaître l'existence de deux modalités du «Bien» hétérogènes et insister sur le fait qu'elles ne sont pas équivalentes constituent des éléments importants de l'éthique déontologique.

Les propos de Mencius sur la «dimension intérieure du sens de l'humain et du sens du juste» (renyi neizai) figurent dans le sixième livre (chapitres 4 et 5) de son œuvre sous le titre «Gaozi, première partie». Ils relatent un débat entre Mencius et Gaozi et leurs disciples respectifs, Gongdouzi et Mengjizi. Analyser

52. MacIntyre Alasdair, After Virtue, Notre Dame, University of Notre Dame Press, 1984, p. 221-223.

53. Dans le même ordre d'idées, voir Mencius 6B: 4 .

54. John R. Silber, «The Copernican Revolution in Ethics: The Good Reexamined», in Robert Paul Wolff (éd.), Kant : A Collection of Critical Essays, Notre Dame, University of Notre Dame Press, 1967, p. 278-287. 
en détail les opinions et thèses des protagonistes dépasse le cadre de cet article ${ }^{55}$ et nous nous limiterons donc aux points suivants: «l'extériorité du sens du juste» (yi wai), telle qu'elle est énoncée par Gaozi et Mengjizi, correspond en quelque sorte à une position de «réalisme moral» (moral realism, daode shizailun) selon laquelle la loi morale et les devoirs sont déterminés par un objet ou une réalité objective. En face, l'optique de Mencius et de Gongdouzi est celle d'un «subjectivisme moral» (daode zhutizhuyi) pour lequel la source de la loi et des devoirs, loin d'être déterminée par quelque objet, relève du sujet moral. Si l'on emploie le vocabulaire de Michael Sandel, on peut dire que Mencius et Gongdouzi affirment «la priorité du sujet sur l'objet», ce qui, naturellement, implique une théorie des devoirs.

Confucius n'a pas, comme Mencius, énoncé clairement de thèse sur «la distinction entre le sens du juste et l'intérêt» ou sur «l'intériorité du sens de l'humain et du juste». Néanmoins, si l'on se base sur certains éléments des Entretiens, nous pouvons légitimement avancer qu'il professe également une éthique des devoirs. En dehors du passage dans lequel il explique que «l'homme de bien valorise le sens du juste et l'homme de peu, l'intérêt» (junzi yu yu yi, xiaoren yu yu li, Entretiens, $4: 16$ ), passage que l'on peut comprendre comme étant à l'origine de la distinction entre les deux notions, Confucius livre sa conception de l'éthique des devoirs lorsqu'il répond à Zai Wo sur la question du deuil des trois ans (Entretiens, $17: 21)^{56}$. Dans le système qui prévalait pendant l'antiquité chinoise, la règle était pour l'enfant de suivre une période de deuil de trois ans lors du décès d'un parent. Trouvant ce temps déjà trop long, Zai Wo, un disciple de Confucius, demanda si le deuil pouvait être écourté et s'achever au bout d'un an:

Le deuil de trois ans après le décès d'un parent est déjà trop long. Si l'homme de bien ne s'adonne pas aux rites pendant trois ans, ceux-ci tomberont certainement en désuétude. Et si, pendant la même période, il en vient à ne plus jouer de musique,

55. Pour un commentaire philosophique sur la «dimension intérieure du sens de l'humain et du sens du juste», voir Mou Zongsan, Yuanshan lun (Traité du Bien parfait), Taibei, Taibei xueseheng shuju, 1985, p. 12-19; Euvres complètes de Mou Zongsan, Taibei, Lianjing chuban gongsi, 2003, vol. 22, p. 11-19. Voir également Lee Ming-huei, Rujia yu Kangde (Les confucéens et Kant), Taibei, Lianjing chuban gongsi, 1990. Deux essais de ce volume renvoient à cette question: «Le confucianisme et la morale fondée sur l'autonomie» et «L'éthique de l'autonomie chez Mencius et Kant».

56. Sur les questions éthiques soulevées dans ce passage, je renvoie aux analyses détaillées d'un article antérieur: Lee Ming-huei, «Das Motiv der dreijährigen Trauerzeit in Lunyu 17:21 als ethisches Problem», in Pohl Karl-Heinz/Wippermannn Dorothea (éd.), Brücke zwischen Kulturen. Festschrift für Chiao Wei zum 75. Geburtstag, Münster, Lit. Verlag, 2003, p. 291-308. 
cette pratique sera à coup sûr perdue. En un an, le vieux grain est épuisé, la nouvelle récolte est prête pour la moisson et le cycle des différents bois utilisés pour allumer le feu est arrivé à son terme ${ }^{57}$; oui, une telle période est certainement suffisante.

Le premier argument de Zai Wo, qui porte sur les rites et la musique, correspond en quelque sorte à ce que l'on appellerait aujourd'hui un point de vue «conséquentialiste» (houguolun, consequentialism). Le second évoque une perspective téléologique (mudilun) qui requiert quelques précisions supplémentaires. En Chine du Nord, il y avait une récolte de riz par an. De plus, dans l'Antiquité, on forait des essences de bois différentes à chaque saison pour allumer le feu. Zai Wo recourt donc à travers ces deux exemples à la norme de ce qui «est» (shiran, en l'occurrence le cycle naturel d'un an du grain ou des essences de bois) afin d'expliquer ce qui «doit être» (yingran) en matière morale (c'est-àdire sur la question du deuil). Autrement dit, il s'agit là d'une sorte de réalisme moral qui ouvre la voie à une téléologie. Cependant, Confucius s'oppose à de telles justifications de la durée du deuil et invite plutôt Zai Wo à s’interroger sur ce qui constitue un réel apaisement intérieur. En procédant ainsi, il replace alors le deuil dans le cadre d'une exigence morale intérieure. Ce passage s'inscrit dans la droite ligne de son idée suivant laquelle le sens de l'humain (ren) est à l'origine du sens des rites $(l i)^{58}$. Si nous recourons à une terminologie moderne, il est possible d'affirmer que la position de Confucius incarne sans aucun doute une sorte de «subjectivisme moral» comportant une théorie des devoirs. Nous avons vu précédemment que dans la philosophie de Kant et de Rawls ces trois éléments que sont l'éthique déontologique, le subjectivisme moral et «la priorité du juste sur le bien » entretiennent une relation logique dont les pensées de Confucius ou de Mencius témoignent aussi pleinement.

Poursuivons notre réflexion en évoquant la conception du Moi chez Confucius et Mencius. Indiquons tout d'abord que, si les idées d'une «connaissance et d'une capacité morales innées » (liangzhi, liangneng) qui dérivent de la théorie mencienne de la «bonté de la nature humaine» (xing shan shuo) s'apparentent à la Bonne volonté kantienne (shan de yizhi, der gute Wille), elles correspondent alors aussi à une sorte de sujet transcendantal (xianyan zhuti). Pour Mencius, «la raison pour laquelle les hommes, sans même apprendre, sont dotés d'une capacité morale tient au caractère inné de cette dernière ; la raison pour laquelle,

57. Nous nous basons ici sur le commentaire de Zhu Xi pour rendre ce passage des Entretiens de Confucius compréhensible. Un type de bois différent était requis à chaque saison pour allumer le feu par friction. Zhu Xi, Si shu zhang ju ji zhu, Zhonghua shuju, 1996, p. 180-181. N.d.T.

58. Confucius de dire: «Si l'homme était dépourvu du sens de l'humain, comment les rites seraient-ils possibles?»(Entretiens, $3: 3$ ). 
sans même réfléchir, ils sont dotés d'une connaissance morale, tient au caractère inné de cette dernière » (Mencius, 7A 17). Il apparaît clairement que ces idées d'une connaissance et d'une capacité morale innées ne sont nullement produites par la société. Néanmoins, elles peuvent - voire même doivent être développées progressivement dans le processus de socialisation (shehuihua) [de l'individu]. Dans le passage précité, Mencius prend l'exemple de Shun ${ }^{59}$ : «Quand Shun habitait au fond des montagnes, au milieu des arbres et des rochers, évoluant parmi les daims et les sangliers, ce qui le distinguait des rudes habitants de ces contrées ne tenait-il pas à presque rien? Quand il entendait ou voyait la bonté d'une parole ou d'un acte, il était alors comme un fleuve débordant que rien ne pouvait arrêter». Ce passage signifie que le processus de socialisation («entendre ou voir la bonté d'une parole ou d'un acte»), s'il n'est pas proprement ce qui constitue la capacité et la connaissance morale innées, n'en constitue pas moins l'occasion de leur déploiement. Confucius n'a quant à lui pas explicitement formulé une théorie suivant laquelle la nature humaine serait bonne. Néanmoins, la réponse qu'il apporte à Zaiwo sur la question du deuil ou sa position sur le sens de l'humain (ren) décrit comme source des rites $(l i)$ nous permettent d'avancer les éléments suivants: Confucius est convaincu que les hommes possèdent un sens de l'humain intrinsèque (renxin zhi ren) qui, manifesté dans les rites, doit être la base des normes sociales. Sa position ne diffère là nullement de celle de Mencius. Dans leurs théories des devoirs ou leur conception du Sujet, les confucéens contemporains sont leurs héritiers.

Si nous essayons de réfléchir à ce que peut être une position confucéenne (classique ou contemporaine) par rapport à ces débats entre communautariens et libéraux, nous aboutissons à un constat assez singulier: les bases éthiques du confucianisme ainsi que sa conception du Sujet le rapprochent à certains égards du libéralisme. Néanmoins, c'est au communautarisme qu'il s'apparente le plus si l'on prend en compte d'une part le rapport de l'individu à la communauté et, d'autre part, sa conception de la tradition. Sur la question de la liberté, il manquait au confucianisme classique un versant «négatif», ce que lui ont apporté les confucéens contemporains, tout en affirmant aussi la liberté positive. Tout cela me porte à dire que, classiques ou contemporains, les confucéens se situent entre communautariens et libéraux ou plutôt, présentent un troisième modèle.

59. L'un des Cinq Souverains avec lesquels commence l'histoire chinoise dans le discours traditionnel, Shun, est considéré comme un parangon de vertu. N.d.T. 


\section{Conclusion : ce que la réalisation d'une philosophie politique néo-confucéenne peut signifier aujourd'hui}

Je souhaite pour conclure évoquer l'utilité qu'a pu avoir la pensée politique confucéenne sur le processus de démocratisation de Taiwan. Il me faut préalablement souligner un point: le débat entre libéraux et confucéens a principalement été une affaire académique, sans influence directe sur la politique réelle. Nous pouvons, à titre de comparaison, évoquer l'influence aux États-Unis de la Théorie de la justice de John Rawls. Il est en effet très difficile d'affirmer que cette œuvre a eu une incidence directe sur le combat électoral. De la même façon, on peut avancer que l'influence des néo-confucéens à Taiwan n'a été qu'indirecte.

En 1949, le gouvernement du Kuomintang, qui venait juste d'opérer une retraite vers Taiwan, y avait alors proclamé la loi martiale, instituant toutes sortes d'interdictions, notamment quant à la possibilité de créer de nouveaux partis politiques. À l'époque, hormis le Kuomintang, deux autres partis s'étaient aussi repliés sur Taiwan: le Parti de la jeunesse chinoise (Zhongguo qingnian dang) et le Parti social démocrate (Minzhu shehui dang). Cependant, les adhérents de ces deux partis étaient très peu nombreux et ils devaient eux-mêmes compter sur le soutien financier du Kuomintang pour subsister, ce qui amena bien des gens à les qualifier de fantoches. En 1960, le diffuseur de Chine libre (Ziyou Zhongguo), Lei Zhen (1897-1979), appela l'opposition et les élites tant indigènes que nouvellement arrivées à constituer un nouveau parti. La réaction des autorités fut vive: Lei Zhen fut jeté en prison et Chine libre cessa de paraître. L'échec de cette tentative fut suivi d'une période assez longue durant laquelle Taiwan resta sous la coupe de ce parti unique qu'était le Kuomintang. Il fallut attendre 1986 et la création par une opposition majoritairement constituée d'élites indigènes et opposée à la loi martiale d'un nouveau parti, le Parti démocratique progressiste (minjindang, DPP), pour que cesse cette situation. Cette fois, le Kuomintang décida de ne pas interdire ce nouveau parti et choisit de s'adapter à la volonté du peuple pour, l'année suivante, mettre fin à la loi martiale. Taiwan commença alors son chemin vers la démocratie. En 2000, le DPP sortit victorieux du scrutin présidentiel, ce qui permit la première alternance, restructura la politique taiwanaise autour de deux grands partis, et renforça la jeune démocratie. Dans l'entreprise de constitution d'un parti d'opposition, les élites locales (par opposition aux Chinois arrivés du continent en 1949) et le mouvement dit «d'indigénisation» (bentuhua) jouèrent un rôle moteur; le libéralisme accompagna cette transformation. Cependant, il est bien ironique de constater que le succès du DPP s'accompagna d'une montée du populisme (mincuizhuyi) qui marginalisa progressivement le camp des libéraux, jusqu'à les évincer presque totalement. 
Dans de telles circonstances, la voix des confucéens dans le processus de démocratisation ne se fit pas vraiment entendre. De surcroît, en affirmant la valeur de la tradition culturelle chinoise, ils pouvaient donner l'impression de faire écho à la politique éducative du Kuomintang mise en œuvre au nom de la tradition confucéenne. Dès lors, certains critiquèrent les confucéens pour leur apathie dans le processus de démocratisation ou allèrent même jusqu'à dire qu'ils lui faisaient obstacle. Des telles critiques sont injustes à mon sens. En effet, la démocratie promue par les confucéens n'était pas une démocratie autoritaire (comme ce que l'on peut voir à Singpapour), mais un véritable modèle de démocratie parlementaire. Zhang Junmai fut d'ailleurs l'un des rédacteurs de l'ébauche de la constitution de la République de Chine (Zhonghua minguo xianfa) promulguée en 1946 à Nankin et qui est toujours en vigueur à Taiwan. Certes, cette constitution fut largement neutralisée du fait de la loi martiale décrétée par le Kuomintang, mais il s'agissait sur le fond d'un texte complètement démocratique. Après 1949, Zhang Junmai resta formellement le président du parti social-démocrate, mais s'étant opposé à la politique dictatoriale de Chiang Kai-shek, il fut longtemps amené à résider à l'étranger où il s'occupa de promouvoir la philosophie confucéenne. Xu Fuguan écrivit pendant longtemps des articles dans les journaux hongkongais qui, en plus de critiquer le Parti communiste, étaient aussi souvent féroces à l'encontre du Kuomintang et soutenaient l'opposition taiwainaise. Ultérieurement, ses prises de position offensèrent la bureaucratie du Kuomintang, ce qui conduisit en 1966 à la cessation de parution de Tribune démocratique. Ces exemples montrent que dénoncer l'apathie des confucéens dans le processus de démocratisation de Taiwan n'est pas chose juste.

Avec la chute de l'empire en 1911, la tradition confucéenne perdit sa place comme idéologie d'État. Progressivement, le Parti communiste chinois lui substitua le marxisme, lequel incarna alors l'orthodoxie. Avec la politique d'ouverture graduelle des années 1980, tant le monde académique que, plus généralement, la société, retrouvèrent vite un intérêt pour la tradition chinoise et le confucianisme. On assista alors à un phénomène qualifié de «fièvre confucéenne» $(\text { ruxue re })^{60}$. À Taiwan, le DPP arriva au pouvoir en 2000 et s'efforça de promouvoir une politique de «désinisation» (qu zhongguohua), mais la société

60. Littéralement, la «fièvre des études confucéennes». Il s'agit d'un engouement qui prend surtout pour foyer le monde académique. Cette situation diffère de celle que l'on observe dans les années 2000, où le confucianisme est plus largement redécouvert, à des degrés très divers, par une certaine partie de la population. N.d.T. Voir Lee Ming-huei, «Das Konfuzianismus-Fieber im heutigen China», in Ralf Moritz et Lee Ming-huei (éd.), Der Konfuzianismus : Ursprünge - Entwicklungen - Perspektiven, Leipzig, Leipzig Universitätsverlag, 1998, p. 235-248. 
taiwanaise n'en préserva pas moins une culture traditionnelle chinoise profonde. Ces dernières années, bon nombre de collègues de Chine populaire en visite à Taiwan me firent remarquer combien il était pour eux manifeste que l'île avait conservé une culture traditionnelle qui la rendait encore plus «chinoise» que le continent. Il ne fait dès lors pas sens d'imaginer que le processus de démocratisation ait pu et puisse encore avoir lieu à Taiwan sans prendre en compte les relations entre démocratie et culture traditionnelle (avec, au premier chef, le confucianisme). Le problème du lien entre tradition confucéenne et système démocratique ne relève pas simplement de la psychologie ou de l'estime que se porte à elle-même la nation, mais elle revêt un sens beaucoup plus concret. Le sociologue Ambrose Y.C.King va également dans ce sens quand il estime qu'un confucianisme reconstruit peut se suturer au système démocratique, donnant alors naissance à un «confucianisme démocratique» (minzhu de rujia), qu'il distingue d'une «démocratie confucéenne» (rujia minzhu). Dans le premier cas, l'élément prépondérant est la démocratie, à laquelle vient s'apparier le confucianisme; dans le second cas, l'élément prépondérant est le confucianisme, qui vient donner un contenu au type même de politique démocratique ${ }^{61}$.

D'un point de vue théorique, les confucéens contemporains réaffirment donc sans cesse qu'il n'y a pas de contradiction essentielle entre une politique démocratique et la tradition culturelle chinoise. Il est difficile de mesurer l'influence indirecte d'une telle position sur les élites taiwanaises. Dans le processus de démocratisation taiwanais, nul n'a utilisé le prétexte de «circonstances nationales » (guoqing) différentes pour refuser un système politique de type occidental, sur le mode de ce que l'on a pu voir à Singapour avec Lee Kwan Yew ou en Malaisie avec Mahathir (nous pensons là au débat sur les «valeurs asiatiques »). Comparé à l'opposition radicale de l'Islam fondamentaliste visà-vis du système démocratique, le processus de démocratisation de Taiwan n'a pas connu de révolution ni d'éruption de violence de grande ampleur; il s'est effectué relativement en douceur. À cet égard, il semble que la position politique très ferme des confucéens contemporains [sur la compatibilité entre la démocratie et la culture traditionnelle] ait joué un rôle très positif. Les conceptions politiques des confucéens n'ont peut-être pas été vraiment prises en compte par les élites politiques libérales ou issues du «mouvement d'indigénisation », mais,

61. King Ambrose Y.C. : «Confucianism, Modernity and Asian Democracy », in Bontekoe Ron et Stepaniants Marietta (éd.), Justice and Democracy: Cross-Cultural Perspectives, Honolulu, University of Hawaii Press, 1997, p.174 sq. Pour mieux prendre la mesure de l'écart qui sépare, pour Ambrose Y.C.King, un «confucianisme démocratique» d'une «démocratie confucéenne», on peut songer à la distance qui sépare une démocratie libérale d'une démocratie populaire (cette comparaison n'est ici opérée que pour prendre conscience de cet écart, elle ne porte pas sur le fond). N.d.T. 
en filigrane, elles ont eu certainement une influence sur le personnel politique du Kuomintang. Elles ont en effet fait comprendre que la démocratisation était la voie à emprunter pour Taiwan et, ce faisant, elles ont contribué à désamorcer les oppositions radicales. Récemment, les «nouveaux confucéens de Chine continentale», représentés notamment par Jiang Qing, ont fait l'objet de l'attention du monde académique occidental ${ }^{62}$. Ce que prône Jiang Qing, c'est de remplacer le marxisme par le confucianisme et il s'oppose à l'adoption par la Chine d'une démocratie qui serait pour lui synonyme de capitulation face à la culture occidentale. Je pense que le fait que ce type de «fondamentalisme confucéen» ne soit jamais apparu à Taiwan nous permet en réalité de mesurer la contribution cachée des nouveaux confucéens à la démocratisation de l'île.

62. Jiang Qing était auparavant professeur à l'Institut d'administration de Shenzhen. Il a pris une retraite anticipée en 2001 pour établir l'académie Yangming (le terme exact que nous traduison par «académie» n'est pas shuyuan, mais jingshe) à Longchang (province du Guizhou, district de Xiuwen), à l'endroit même où Wang Yanming, la figure majeure du confucianisme Ming, avait «pris conscience de la Voie» (wu dao). 


\section{Glossaire}

bentuhua 本土化

chao chuantongxing 超傳統性

Chen Duxiu 陳獨秀

daode faze youxian yu shan 道德法則優先於善

daode shizailun 道德實在論

daode zhutizhuyi 道德主體主義

dexing 德性

dexing 德行

diciyuan de chuantong 低次元的傳統

e 惡

Feng Youlan 馮友蘭

gaociyuan de chuantong 高次元的傳統

Gaozi 告子

geren de ziwo shixian 個人的自我實現

gerenzhuyi 個人主義

getihua de 個體化的

gexing 個性

Gongdouzi 公都子

gonghezhuyi 共和主義

gonglizhuyi 功利主義

gongmin de dexing 公民的德行

guojiao 國教

guojia ziyou 國家自由

guoqing 國情

hanhun 含混

heli de jingyanlun zhi guizhun 合理的經驗論之規准

$\mathrm{Hu}$ Shi 胡適

Huaqiao ribao 華僑日報

Jiang Qing 蔣慶

jitizhuyi 集體主義

jiji ziyou 積極自由

jihui gainian 機會概念

jingshe 精舍

jiquan minzhu 集權民主

Jie Ni 桀溺

Jin Yuelin 金岳霖

jingshen zhuti 精神主體

Kang Youwei 康有為

Lei Zhen 雷震

li 禮

Liang Hui Wang 梁惠王 
liangneng 良能

Liang Shuming 梁漱溟

liangzhi 良知

Lun chuantong 論傳統

lunli 倫理

Mei Yunli 梅藴理

Mencius 孟子

Mengjizi 孟季子

mincuizhuyi 民粹主義

Minjindang 民進黨

Minzhu Pinglun 民主評論

minzhu de rujia 民主的儒家

Minzhu shehui dang 民主社會黨

minzu 民族

mudilun 目的論

mudi wangguo 目的王國

Mou Zongsan 牟宗三

neizai yaoqiu 內在要求

pianmian de 片面的

Qian $\mathrm{Mu}$ 錢穆

qingxuzhuyi daodeguan 情緒主義道德觀

qu zhongguohua 去中國化

ren 仁

rengezhuyi 人格主義

renxin zhi ren 人心之仁

ren yi nei zai 仁義內在

rujia minzhu 儒家民主

ruxue re 儒學熱

shehuihua 社會化

shan 善

shan de yizhi 善的意志

shan zhi yizhixing 善之一致性

shiran 實然

shizhi ziyou 實質自由

shequn 社群

shequn yishi 社群意識

shuyuan 書院

Shun 舜

Tang Junyi 唐君毅

weixinlun de yiyuanlun 唯心論的一元論

Wen 文

$\mathrm{Wu}$ 武

wu dao 悟道

wu zhi zhi mou 無知之謀 
xianyan zhuti 先驗主體 xian yi hou li 先義後利 xiaoji ziyou 消極自由 xin minzhu 新民主 xingshi ziyou 形式自由 xing shan shuo 性善說

Xiong Shili 熊十力

Xu Fuguan 徐復觀

Yao 堯

yi li zhi bian 義利之辨 yiwulun 義務論

yiwulun ziyouzhuyi 義務論自由主義

yinguolü 因果律

Yin Haiguang 殷海光

yingran 應然

yuanchu zhuangtai 原初狀態

yuanzilun 原子論

yushe 預設

yunzuo gainian 運作概念

Zaisheng 再生

Zai Wo 宰我

Zhang Foquan 張佛泉

Zhang Ju 長沮

Zhang Junmai 張君勱

Zhang Qiyun 張其昀

zhengyi ji gongping 正義即公平

zhengque youxian yu shan 正確優先於善

zhengzhi shequn 政治社群

zhonghua minguo xianfa 中華民國憲法

Zhongguo qingnian dang 中國青年黨

zhongxue wei ti, xixue wei yong 中學為體, 西學為用

zijue de 自覺的

Zilu 子路

Ziyou Zhongguo 自由中國

ziyou minzhu 自由民主

zizuo zhuzai 自作主宰

ziwoguan 自我觀

ziwo youxian yu mudi 自我優先於目的

ziwo rentong 自我認同

ziyou shehui 自由社會 